\title{
Galvinoxyl monolayers on Au(111) studied by STM, EPR, and cyclic voltammetry
}

\author{
Natascha Niermann,,${ }^{1,2}$ Tesfaye Hailu Degefa, ${ }^{3}$ Lorenz Walder, ${ }^{3}$ Vitali Zielke, ${ }^{4}$ Heinz-Jürgen Steinhoff, ${ }^{4}$ \\ Jens Onsgaard, ${ }^{5}$ and Sylvia Speller ${ }^{1, *}$ \\ ${ }^{1}$ Institute for Molecules and Materials, Radboud Universiteit Nijmegen, 6525ED Nijmegen, The Netherlands \\ ${ }^{2}$ Fachbereich Physik, Universität Osnabrück, 49069 Osnabrück, Germany \\ ${ }^{3}$ Organische Chemie und Molekulare Elektrochemie, Institut für Chemie, Universität Osnabrück, 49069 Osnabrück, Germany \\ ${ }^{4}$ Makromolekülstruktur, Fachbereich Physik, Universität Osnabrück, 49069 Osnabrück, Germany \\ ${ }^{5}$ Department of Physics and Nanotechnology, Aalborg University, Pontoppidanstraede 103, DK-9220, Aalborg East, Denmark
}

(Received 22 September 2005; revised manuscript received 10 June 2006; published 14 December 2006)

\begin{abstract}
Galvinoxyl layers on $\mathrm{Au}(111)$ have been studied by scanning tunnelling microscopy (STM), electron paramagnetic resonance (EPR), and cyclic voltammetry $(\mathrm{CV})$. We observe two phases: configuration I having a molecular density of $1.57 \pm 0.16 \times 10^{-10} \mathrm{~mol} / \mathrm{cm}^{2}$ and a rectangular lattice $(15 \AA$ by $7 \AA$ ) observed at room temperature and down to $140 \mathrm{~K}$; configuration II with a slightly smaller molecular density of $1.37 \pm 0.14$ $\times 10^{-10} \mathrm{~mol} / \mathrm{cm}^{2}$ and oblique cells $(22.5 \AA$ by $5.4 \AA)$ arranged alternatingly in stacks yielding a molecular layer with lower symmetry and comparatively large crystallographic unit cell. The latter is only observed upon cooling down to $40 \mathrm{~K}$ and subsequent annealing to room temperature. For both assemblies typical domain sizes in the range of $100 \mathrm{~nm}$ have been found. The EPR results confirm that the radical character is preserved upon adsorption and that the intermolecular distance is smaller than $11 \AA$. The interaction between the overlapping singly occupied spin orbitals is high, indicating no participation of the unpaired electron in the binding to the surface or laterally between neighboring radicals. The average surface concentration deduced from CV measurements is in excellent agreement with the surface coverages deduced from STM topographies. In aqueous electrolyte the adsorbate undergoes a one-electron oxidation with concomitant loss of a proton as determined from oxidation potential vs $p \mathrm{H}$ curves in a similar fashion as known for the free radical in solution. This indicates no dramatic change of the electronic properties of the radical upon adsorption. Structure models are proposed with molecules standing upright like "bicycles in rows."
\end{abstract}

DOI: 10.1103/PhysRevB.74.235424 PACS number(s): 68.43. $-\mathrm{h}, 68.37 .-\mathrm{d}, 75.70 .-\mathrm{i}$, 82.45. $-\mathrm{h}$

\section{INTRODUCTION}

Interestingly, purely organic ferromagnets show long range magnetic order in the crystalline phase. Organic molecular magnets are in many respects superior to conventional magnets because they are comparably small and light, and chemically easy to control. ${ }^{1}$ Galvinoxyl is one of the most promising radicals because it is a persistent and stable radical exhibiting a ferromagnetic phase at room temperature. ${ }^{2,3}$ Ferromagnetic nanostructures are expected to form an increasingly important class of materials in future technologies and the study of ferromagnetic layers of radicals is therefore intriguing. We investigate the structure and the electronic properties of galvinoxyl adsorbed on $\mathrm{Au}(111)$.

In principle every atom or molecule with an unpaired electron is predefined as a radical. But only few of them are stable and persistent at ambient conditions and upon adsorption. For stable radicals, to the best of our knowledge only nine studies of nine different radical assemblies, respectively, aggregate structures on surfaces investigated by STM are published. ${ }^{4-12}$ Combined EPR and STM was used to detect spin specific signals through the tip by applying a magnetic field and a microwave to get STM-EPR spectra of $g$-bisdiphenylene- $b$-phenylallyl (BDPA) clusters containing free radicals. ${ }^{4}$ The high spatial resolution of STM together with the electronic spin sensitivity of electron-spin resonance showed the presence of localized spins in BDPA on a HOPG (highly oriented pyrolitic graphite) surface. Three other studies describe the adsorption of TEMPO $(2,2,6,6-$ tetramethylpiperidin-1-oxy) on $\mathrm{Si}(100)$ and $\mathrm{Si}(111)$. The stable and free radical is reduced and connected by dangling bonds to the surface and investigated by scanning tunnelling microscopy with varying tunnelling currents. ${ }^{5,6}$ The most recent study combines STM measurements and computational modelling of TEMPO on $\mathrm{Si}(100) .{ }^{7}$ A strong bias dependence in the topography of isolated molecules was observed in the range of -2 to $+2.5 \mathrm{~V}$. The computational method could confirm the binding mechanism to the surface. STM and LEED study indicate that methoxy, ethoxy, phenoxy and 1-propoxy adsorb into $p(2 \times 2)$-S vacancies on a sulfurcovered $\mathrm{Ni}(100)$ surface. ${ }^{8}$ It has been shown by STM that methyl radicals adsorb as two-dimensional (2D) islands on $\mathrm{Cu}(111)$ up to a coverage as a saturation monolayer. ${ }^{9}$ For two important radical species, i.e., methyl $\left(\mathrm{CH}_{3}\right)$ and methylnitrene $\left(\mathrm{NCH}_{3}\right)$ on $\mathrm{Cu}(111)$ and $\mathrm{Cu}(110)$ high-resoluted STM images have been obtained and plausible adsorption models have been derived..$^{10}$ By using the reduced thermal mobility of molecular radicals on surfaces, it was demonstrated that the cyclopentadienyl radical can be readily manipulated lateral to the STM tip motion and associatively desorbed with a STM tip at room temperature. ${ }^{11,12}$ Some of the radicals described were reduced by binding to the surface.

In the wide field of radical research based on STM investigations we refer to several representative examples. One of the best examples is pure nitrogen, which is adsorbed on a $\mathrm{Si}(100)$ surface in UHV. Linear defects perpendicular to dimer rows found with STM and scanning tunnelling spectroscopy (STS) revealed that the initial nitridation reaction occurs preferentially at the backbonds of surface $\mathrm{Si}$ atoms. ${ }^{13,14}$ Another chemically very simple radical which is 
nitric oxide (NO) was adsorbed to a $\mathrm{Cu}(110)$ surface. The molecule splits off and $\mathrm{N}$ - and O-induced islands are detected. ${ }^{15}$ In the case of NO two different adsorption structures on $\mathrm{Pt}(111)$ observed by STM have been reported. ${ }^{16} \mathrm{Ad}-$ ditionally the chemisorption of $\mathrm{NO}$ on a flat stepped $\mathrm{Ru}(0001)$ surface was calculated using density functional theory. ${ }^{17}$

For organic molecules deposited on surfaces we refer to the review papers of Blundell et al., ${ }^{18}$ Schreiber, ${ }^{1}$ and Kinoshita. ${ }^{19}$

The study of the magnetic behavior of organic radicals has started already in 1957 with the interest on galvinoxyl (2,6-di-tert-butyl-4-(3,5-di-tert-butyl-4-oxycyclohexa-2,5-dienyliden-emethyl)-phenoxy. ${ }^{20}$ The molecule was first named after its discoverer "Coppinger-radical," obviously his first name Galvin provided the contemporary name. Galvinoxyl is considered most interesting as the compound in the solid phase passes a first order magnetic phase transition at $85 \mathrm{~K}$ from ferromagnetic above, to antiferromagnetic below this temperature. ${ }^{3,18}$

Several studies have been presented on crystalline galvinoxyl. The EPR spectra of the polycrystalline specimen shows a flat-topped single peak due to the anisotropy of packing of the individual crystallites. ${ }^{21}$ The nature of the spin exchange interaction between the unpaired electrons has been described using the band model. ${ }^{22}$ In order to understand the mechanism of the magnetic interactions, ferromagnetic properties like spin population, spin arrangement and the spontaneous magnetic moment per molecule have been calculated by $a b$ initio studies. ${ }^{23}$ It was found that the total DOS below the Fermi level exhibits sharp peaks, i.e., energy bands below the Fermi level are narrow and flat. The magnetic properties of a mixed crystal of the galvinoxyl radical and its precursory closed shell compound have been studied. ${ }^{24}$ From these measurements it was concluded that the ferromagnetic order, which passes a transition at $85 \mathrm{~K}$ in pure galvinoxyl, is maintained down to $2 \mathrm{~K}$ in mixed crystals. Though, there is no clear statement for the Curie temperature of galvinoxyl. Many of the conditions necessary to be an organic ferromagnet above the $85 \mathrm{~K}$ transition temperature are fulfilled.

Inside the galvinoxyl crystal the almost planar radical molecules are arranged in different forms of alternating stacks. ${ }^{25}$ Different configurations were assumed and all of them have similar smallest intermolecular distances. The oxyphenyl residues are twisted relative to each other by about 12 degrees. Therefore, in all our models we presume a planar structure for the molecule as an approximation. The stacks of the molecular crystals are found to be $\sim 13.9 \AA$ wide with an intermolecular distance inside these stacks along the $c$ axis of roughly $0.65 \mathrm{~nm}^{3}$. Furthermore, the distance of about $3.71 \AA$ between the carbon atoms of two adjacent molecules ${ }^{22}$ is relatively high compared to distances found in stacks of other radicals. Nevertheless, the typical $\pi-\pi$ interaction is presumed.

Attempts to obtain the crystal structure of pure galvinoxyl (purity $>90 \%$ ) below $85 \mathrm{~K}$ failed due to disintegration of the crystals while the samples were cooled through the transition temperature. ${ }^{26}$ The coupling between the single electron spins exhibits a large intramolecular exchange which leads to a high spin polarization.
Experimental studies of crystalline galvinoxyl showed that the magnetic properties do vary with temperature. The high temperature susceptibility is well reproduced by the one-dimensional ferromagnetic Heisenberg model and follows the Curie-Weiss law with a positive Weiss constant of $19 \mathrm{~K}$ above $85 \mathrm{~K}^{18}$ Therefore this radical is considered to have ferromagnetic intermolecular interaction, while most of the organic radicals rather have antiferromagnetic interaction. Galvinoxyl received additional attention due to the phase transition at $85 \mathrm{~K}$. Slightly different behavior has been reported around $85 \mathrm{~K}$ and the paramagnetic susceptibility is supposed to be weaker below the transition temperature of $85 \mathrm{~K}$ down to $2 \mathrm{~K}^{24}$ Antiferromagnetic interactions have been suggested with a negative Weiss constant in this temperature regime. ${ }^{18}$ Small impurities seem to suppress this phase. $^{24}$

Galvinoxyl is a promising candidate to stay stable on an metallic surface as two dimensional array, the free radical is not changing its oxidation state easily by exposure to oxygen or light. Stability of radicals is generally related to a combination of odd electron delocalization and steric hindrance preventing dimerization, referring to the ortho tert-butyl groups in the case of galvinoxyl. Galvinoxyl is known to be a very reliable radical scavenger. ${ }^{27,28}$

\section{EXPERIMENT}

\section{A. Methods}

The STM experiments were carried out in an ultrahigh vacuum (UHV) system with separate analysis and preparation chamber. Most of the STM measurements were done with an Omicron VT (variable temperature) Micro-SPM (scanning probe microscope) and an STM I using tungsten tips. Both systems were very well calibrated and permanently checked for drift control during the measurements. Calibration was done using atomically resolved $\mathrm{Au}(111)$ surfaces and steps. As the lateral calibration factor depends on frame size and history, calibration was refined using the $\mathrm{Au}(111)$ herringbone reconstruction. This indicates that for midsize frames $(\sim 100 \mathrm{~nm})$ the $x$ and $y$ scale should be contracted by $10 \%$. We show the original data.

Deposition of the molecules was done from solution using a commercial pulse valve built by Parker Instruments Inc. ${ }^{29}$ It was operated with closure times of $0.5 \mu \mathrm{s}$ up to $25 \mu \mathrm{s}$. For this purpose a home-built supply was used. Further details will be presented in a more technical presentation that will follow later on. ${ }^{30}$ The spray valve was mounted at the inlet chamber. After every pulse we waited to allow the pressure to recover to the $10^{-7}$ mbar region. On top of the valve a small crucible was filled with the molecule solution and was covered by a ceramic cap.

The EPR measurements were performed with a Varian CW Multifrequency X-band $(9.5 \mathrm{GHz}$ ) Spectrometer (Varian E109) equipped with a standard rectangular $\mathrm{TE}_{102}$ resonator (Varian model) described in the technical manual. ${ }^{31}$ The gold samples with the galvinoxyl layer were oriented parallel with respect to the magnetic field lines. A defined amount of the solution in the range of $10^{14}$ molecules was released onto the $\mathrm{Au}(111)$ substrate. Alternatively the gold surface was im- 
mersed inside the molecule solution. The instrument settings were as follows: microwave power $5 \mathrm{~mW}$, time constant (Lock-In integration) $300 \mathrm{~ms}$, modulation amplitude $1 \mathrm{G}, 10$ repeats.

All electrochemical measurements were performed in a three-electrode system under Ar, using a potentiostat PGSTAT 20 from AUTOLAB interfaced with a personal computer running under GPES for Windows, Version 4.9 (ECO Chemie). ${ }^{32}$ Beside the galvinoxyl coated gold electrode (working electrode), a $\mathrm{Ag} / \mathrm{AgCl}$ reference electrode (Methrom, 6.0724.140, separated by a salt bridge containing the electrolyte of the measuring compartment), and a platinum wire as the counter electrode were used. As supporting electrolyte we used $0.1 \mathrm{M} \mathrm{Na}_{2} \mathrm{HPO}_{4}$. The $p \mathrm{H}$ of the solution was adjusted to the desired values with $0.1 \mathrm{M} \mathrm{NaOH}$ or $\mathrm{HCl}$. Unless otherwise indicated, the $\mathrm{CV}$ was performed at $100 \mathrm{mV} / \mathrm{s}$. The surface concentration was calculated from $\mathrm{CV}$ using the surface beyond the voltammogram corrected for the capacitive contribution. The $E^{0}$ was calculated from each CV using $E^{0}=\left(E_{p a}+E_{p c}\right) / 2$.

\section{B. Sample preparation}

$\mathrm{Au}(111)$ layers with $100 \mathrm{~nm}$ to $200 \mathrm{~nm}$ thickness were prepared by Au evaporation from a crucible onto a clean and annealed mica film of $0.49 \mathrm{~cm}^{2}$. The evaporation took place at a pressure of $10^{-7} \mathrm{mbar}$ in a separate high vacuum setup. The samples have been locked into the UHV setup. Standard $\mathrm{Ar}^{+}$, respectively, $\mathrm{Ne}^{+}$sputter $(1 \mathrm{keV}, 2-5 \mu \mathrm{A}, 10 \mathrm{~min})$ and annealing cycles $(550 \mathrm{~K}, 10 \mathrm{~min})$, have been applied to clean and flatten the surface. The free radical galvinoxyl has been solved in pure ethanol. The mm-molar solution was sprayed directly into the vacuum chamber onto the atomically flat $\mathrm{Au}(111)$ surface. Parameters were optimized by subsequent variation depending on the conditions of evaporation, i.e., pumping speed in UHV. The evaporation speed plays a decisive role during deposition because vapor distribution of solvent and radical differ and the molecular layer is affected in the following way: during the deposition procedure the solution cools down, the evaporation profile of the solvent ethanol is different in comparison to the molecule galvinoxyl. Consequently the evaporation speed influences the ratio of galvinoxyl to ethanol adsorbing on the surface. Additionally with lower pumping speed the solution cone opens. After spraying the solution onto the surface the sample was annealed in the UHV to $50{ }^{\circ} \mathrm{C}$ to eliminate the deposited solvent from the surface. This procedure was controlled by a mass spectrometer.

For the experiments with EPR and cyclic voltammetry a different preparation method had to to be used. Under ambient conditions the substrate has been immersed into a $\mathrm{mM}$ solution containing the galvinoxyl molecules solved in ethanol at $323 \mathrm{~K}$, afterwards taken out and dried with pure nitrogen. The compound was bought from Aldrich with a purity of $98 \%$. The concentration inside ethanol solution was varied from $0.2 \mathrm{mM}$ to $1 \mathrm{mM}$.

For the EPR measurements the radical layers were prepared depositing a defined amount of the galvinoxyl solution onto the $\mathrm{Au}(111)$ surfaces using pure ethanol as solvent. If

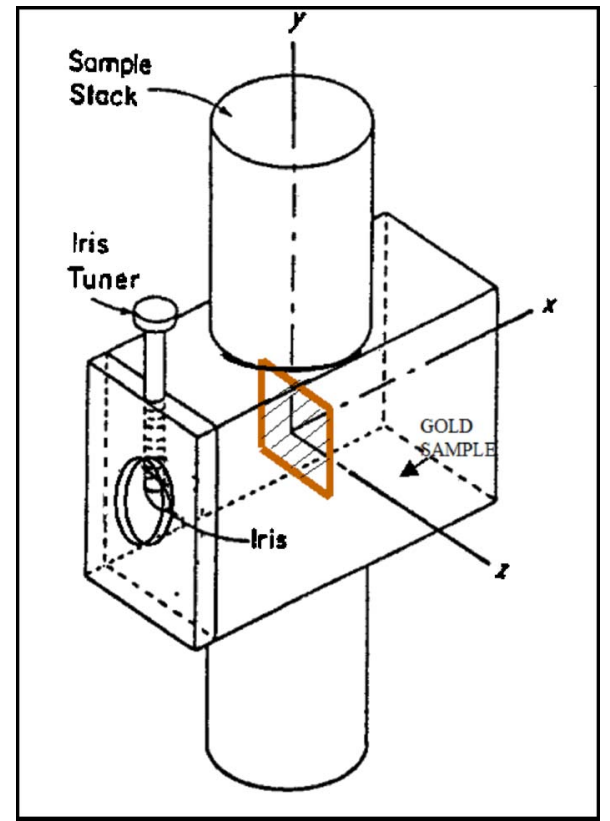

FIG. 1. (Color online) Drawing of the sample position inside the resonator.

not otherwise stated $2 \mu \mathrm{l}$ were administered, taken from a $0.2 \mathrm{mM}$ galvinoxyl solution in ethanol. The sample was placed inside the resonator as shown in Fig. 1 with a selfconstructed plastic holder.

The sample for electrochemical measurements was prepared by exposing freshly cleaned gold disk electrodes (Metrohm, 6.1204.140, $3 \mathrm{~mm}$ diameter) to $1 \mathrm{mM}$ galvinoxyl in ethanol for $24 \mathrm{~h}$. Prior to the sample preparation, the electrodes were polished to a mirror-finish with a wet $\mathrm{Al}_{2} \mathrm{O}_{3}$ slurry on a flat pad (Metrohm, 6.2802.000, grain size $0.3 \mathrm{~m}$ ), rinsed thoroughly with distilled water, cleaned for $2 \mathrm{~min}$ with freshly prepared piranha solution (2:3 mixture of $30 \%$ $\mathrm{H}_{2} \mathrm{O}_{2}$ and concentrated $\mathrm{H}_{2} \mathrm{SO}_{4}$.), rinsed with ethanol followed by distilled water. The coated electrode is then ready for characterization after rinsing with ethanol to remove any physically adsorbed molecules.

\section{Galvinoxyl: structure and spin density of the free molecule}

In Fig. 2 the chemical structure of the molecule is shown.

In order to understand the pattern of closely packed galvinoxyl radicals observed by STM on $\mathrm{Au}(111)$, we start from an isolated molecule modelled using a semiempirical AM1 calculation. ${ }^{33}$ Figure 3 shows a quasi-three-dimensional (3D) view of the molecule. The grids in green (positive) and violet (negative) represent the spin density from the Unrestricted Hartree-Fock (UHF) AM1 calculation. The optimized structure will serve to extract the orientation of the molecules within the stacks on $\mathrm{Au}(111)$ observed in STM topographies.

The spin densities of the galvinoxyl radical optimized in structure demonstrates the large extent of spin density distribution over the entire $\pi$ system. This fact, in combination with the shielding effect of the tert-butyl groups, is the main reason for the stability of the radical. 


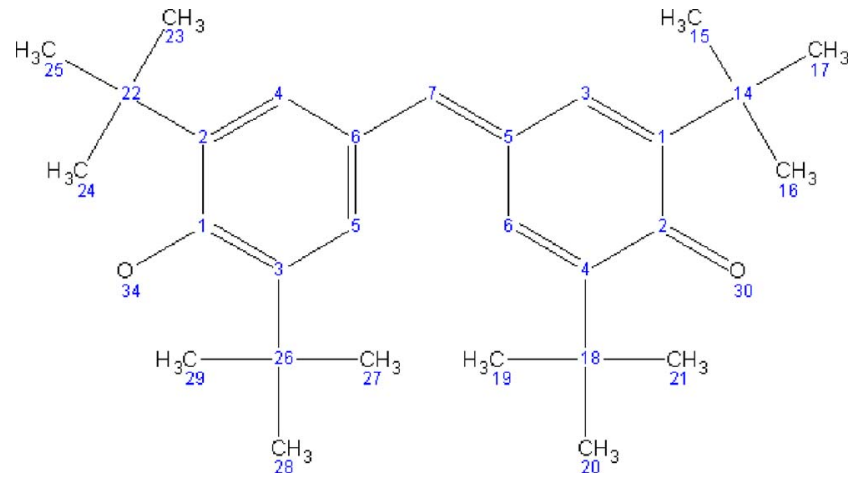

FIG. 2. (Color online) Chemical structure of galvinoxyl with the atoms labelled.

Figure 4 shows the molecular orbital close to the Fermi level, i.e., at $-1.555 \mathrm{eV}$ indicating the regions, which are important for the highest tunnelling probability during STM measurements.

\section{RESULTS AND DISCUSSION}

In this study we investigate experimentally the structural, electronic, magnetic, and chemical characteristics of the selfassembled monolayer.

\section{A. Topography: STM results}

We obtained topographies with submolecular resolution of the monolayer assemblies of galvinoxyl on the Au(111) surface. Two types of patterns were observed. Both configurations show arrays of stacks, built from galvinoxyl molecules. The orientation of the molecule stacks is along the six low-index directions of the $\mathrm{Au}(111)$ surface due to the sixfold symmetry of the well-known $22 \times \sqrt{3}$ reconstruction of $\mathrm{Au}(111){ }^{34,35}$ The combination of theoretical results on the crystalline phase, the observed corrugation in STM topographies, and the voltammetry results allowed us to estimate the molecular density on the surface. We consider two structure models: one with rectangular lattice, consisting of stacked molecules, in parallel aligned and a second more complex

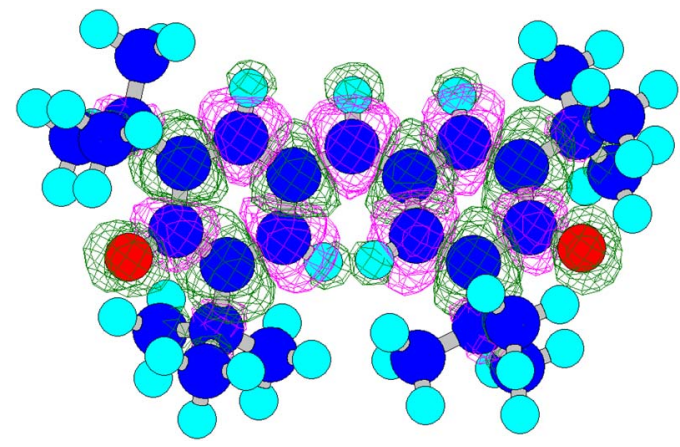

FIG. 3. (Color online) Result of the semiempirical AM1structure calculation, geometry optimization done by Polak-Ribiere (conjugate gradient) (Ref. 33) of the free galvinoxyl radical; violet (negative)-green (positive) lobes illustrate the spin density (contour value, 0.001)
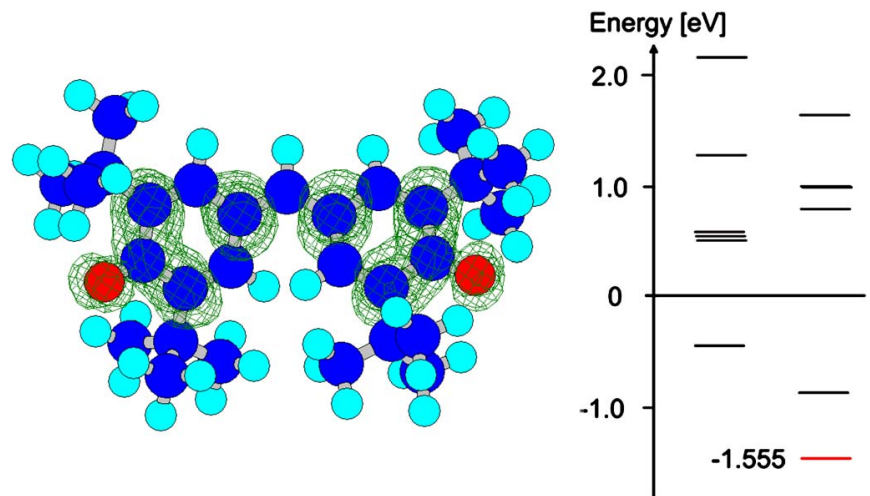

FIG. 4. (Color online) AM1-optimized, geometry optimization by Polak-Ribiere (conjugate gradient) (Ref. 33); molecule with lobes indicating squared coefficients of the orbital close to the Fermi level, at $-1.555 \mathrm{eV}$ with a contour value of 0.0005 (left-hand side); energy levels of the orbitals in the frontier orbital region (right-hand side).

structure with oblique angles. In both cases the double-ring molecule is standing upright on the surface. The two dominating interactions, overlap of $\pi$ orbitals, especially inbetween the carbon rings, and hydrogen bonding, favor the molecules to stand upright like bicycles in a row, and an ordered array is formed. Stacking of aromatic parts of molecules is very common, e.g., shown for porpherins. ${ }^{36}$ The two configurations differ in the angle between the molecules of adjacent stacks. Both configurations of the monolayer assembly have been observed by STM. To check for spinordering in the domains we used antiferromagnetic $\mathrm{MnNi}$ tips. ${ }^{37}$ Antiferromagnetic tips have no stray field that influences the sample magnetism. However, down to $140 \mathrm{~K}$ we found no appreciable difference in topography using these tips. Either the sensitivity, i.e., the spin-polarization of the tip is too $\operatorname{low}^{38}$ or spin-ordering in the aggregate is not sufficiently strong or absent.

The energy scale of the molecular orbital and the tunnelling bias scale must not necessarily coincide. On the one hand, the absolute energies depend on the reference chosen in the theoretical description, and on the other hand, molecular orbitals shift substantially in energy upon physisorption. It is however likely that the singly occupied molecular orbital (SOMO) gets close to the Fermi level and that at low biases, no matter positive or negative, the current passes the SOMO. The topography remains largely unchanged with varying bias in the interval $-2.0 \mathrm{~V}-+2.0 \mathrm{~V}$.

The EPR data confirmed the free radical remaining stable on the surface, i.e., not being reduced. This allows us to conclude that the dominating interaction in the self-assembly can be attributed to the intermolecular forces, and not to the bonding between galvinoxyl molecules and the gold atoms. A dimerization energy of $-51 \mathrm{~kJ} / \mathrm{mol}$ is reported. ${ }^{39}$ It is probable that intermolecular forces lead to dimerization effects already in solution. This is consistent with results on gavinoxyl crystals, ${ }^{25}$ where all structures rely mainly on the interaction between the carbon rings. ${ }^{3}$ Also it has been calculated, that radicals will have preferred adsorption positions on metal surfaces. ${ }^{40}$ The binding to the surface is mainly due to the comparably weak van der Waals forces. 


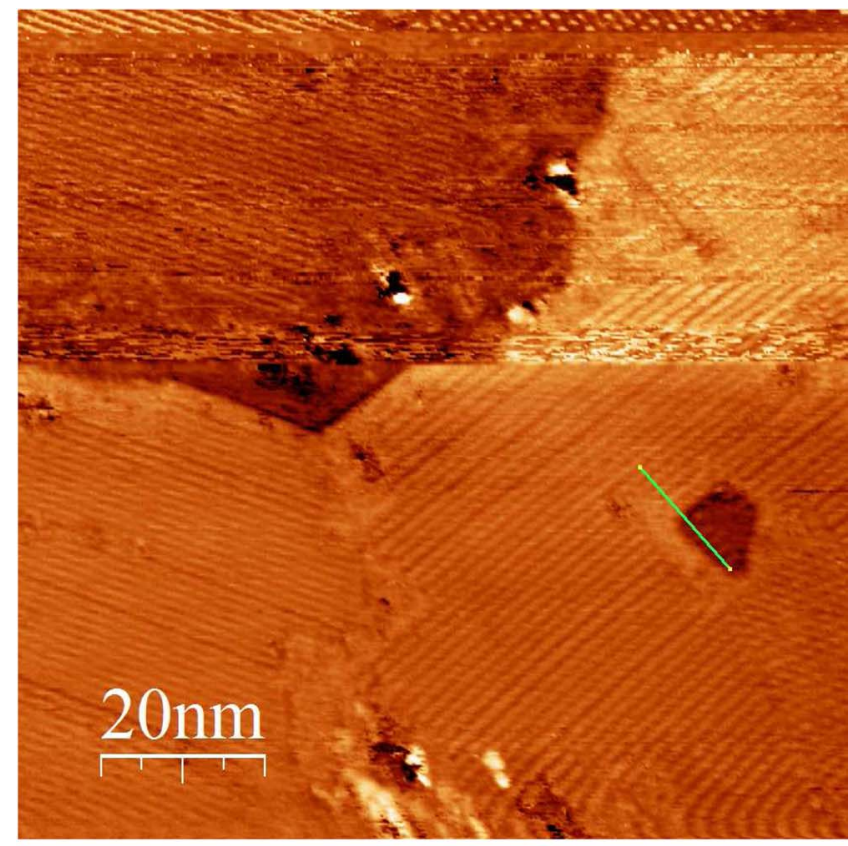

(a) $\mathrm{U}_{\text {gap }}=-1.452 \mathrm{~V}, \mathrm{I}=0.021 \mathrm{nA}$, total height $11.4 \AA$

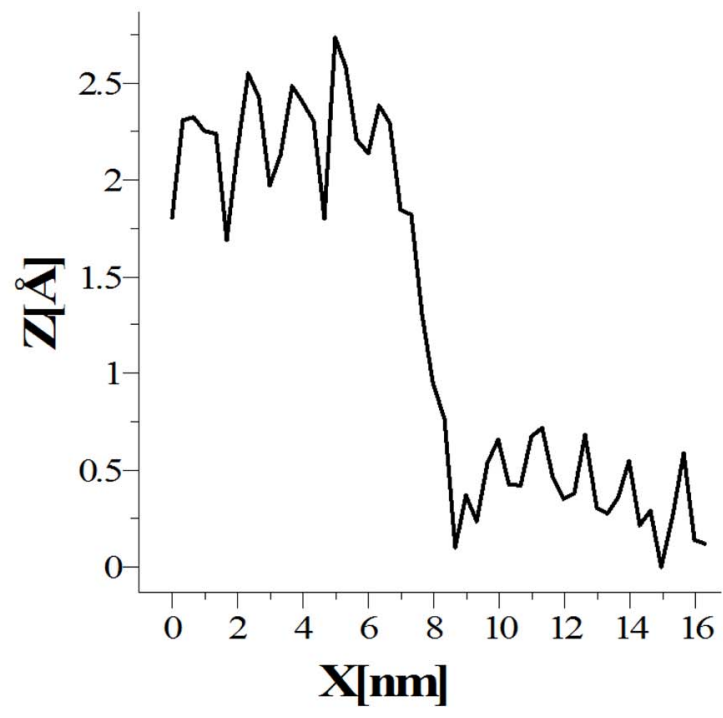

(b) line profile; averaged height difference between plateaus: $2.35 \AA$

FIG. 5. (Color online) STM topography of galvinoxyl on $\mathrm{Au}(111)$ taken at room temperature, sample preparation at room temperature, frame size $100 \mathrm{~nm}$.

\section{Configuration I}

The first configuration has been imaged by STM at room temperature and at $140 \mathrm{~K}$. The deposition took place at room temperature.

Figure 5 shows the topography of galvinoxyl deposited at room temperature. On the terrace characteristic corrugation with an amplitude of $0.8 \AA$ is observed. Straight step edges are as likely as not due to steps on the $\mathrm{Au}(111)$ substrate underneath. Other types of meandered step edges are present. Most significant, a line pattern is observed, that is attributed to the molecular aggregate on the surface. The periodicity of this line pattern is $\simeq 15 \AA$. Along the ridges no corrugation is observed, which can, however, be due to insufficient resolution. A few etching holes can be seen clearly and the line profile shows that the same arrangement of the molecules is present inside the hole like on the rest of the surface, see Fig. 5(b). At room temperature the holes are mobile until the system is stabilized, wandering with speeds of about $5 \mathrm{~nm}$ per frame scan, a frame scan lasts about 2 minutes at this frame size. The height difference between hole and terrace is $2.38 \AA$ and can be attributed to the step height of $\mathrm{Au}(111)$ of $2.35 \AA$, within the experimental error.

The STM topography of the galvinoxyl layer on $\mathrm{Au}(111)$ observed at $140 \mathrm{~K}$ is presented in Fig. 6. Qualitatively the structure does not change regarding the room temperature structure. The same characteristic line patterns with distances between the molecules of $15 \AA$ as at room temperature are observed. In contrast to the room temperature measurement the etching holes appear more sharp. While keeping the sample at low temperature we observed even less etching holes than at room temperature. The comparison of images taken at different times after deposition reveal that the monolayer becomes increasingly more stable inside UHV. This is also true for room temperature but at low temperature this effect is much stronger. The aggregate appeared inert against impurities, it stays nearly at the same condition for several weeks in UHV at a pressure below $1 \times 10^{-10}$ mbar. Most striking is the enhanced contrast of the self-assembly compared to room temperature. The pattern can be imaged at higher tunnelling current without moving around molecules on the surface, this was not possible at room temperature. The different contrast in the middle of the image arises from a change in the tip state, which allows one to resolve a substructure within the lines with a period of $30 \AA$, which is difficult to resolve with the other tip state. The $30 \AA$ are only observed exceptionally upon tip changes, probably a molecule or part of a molecule is picked up. Thus, the same structure is present in both areas, the $15 \AA$ period being identical, but the sensitivity of the tip changed.

A high-resolution STM topography of galvinoxyl layer on $\mathrm{Au}(111)$ is presented in Fig. 7. This image shows a typical zoom into the stripe pattern, that we attribute to molecular stacks. This frame includes two contiguous domains, that enclose an angle of 60 degrees. We concluded that these domains follow the low index directions of the $\mathrm{Au}(111)$ surface. The transition from one domain to the other shows disordered patches of molecules, sometimes forming clusters. The disordered regions shrink upon heating the sample to $333 \mathrm{~K}$. Annealing leads to ordering of the molecular layer while excess molecules desorb. The green bar has a length of $75 \AA$ spanning 5 lines $15 \AA$ wide.

The correlation filtered STM topography of the galvinoxyl layer on $\mathrm{Au}(111)$ is presented in Fig. 8. The filtering allows one to enhance substantially the contrast and thereby to recognize more details of the assembly and the molecules.

A closer view to the filtered data provides intramolecular details that allow us to derive a model. Figure 8(b) shows a rectangular pattern with a basis with twofold rotation symmetry. Intramolecular details become visible. This can however reflect the symmetry of the tip. Taking line profiles in 


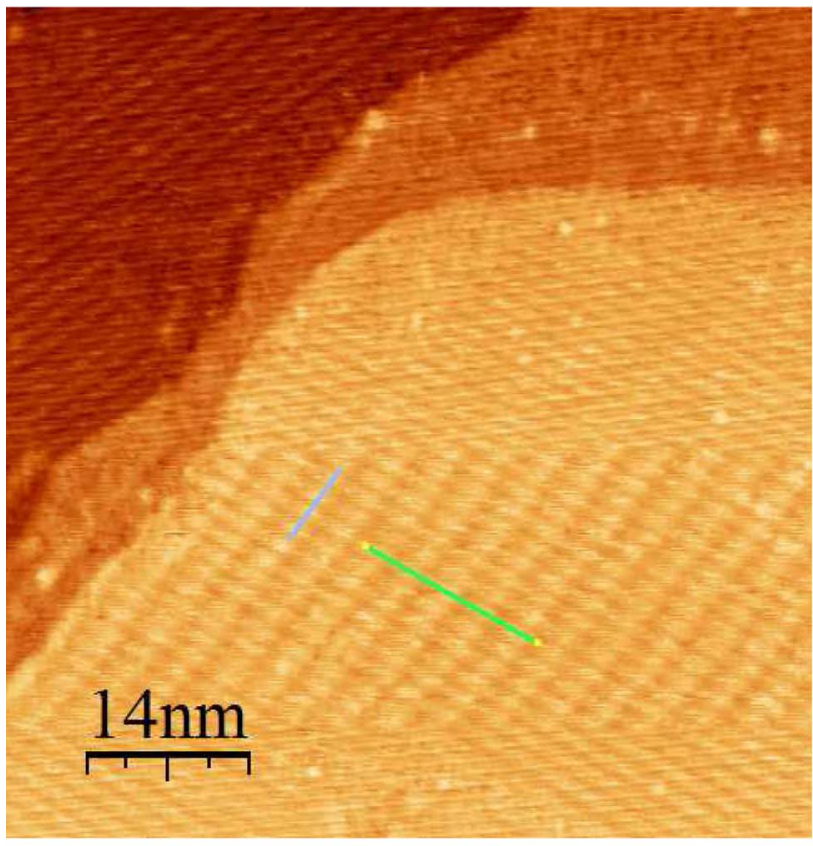

(a) $\mathrm{U}_{\text {gap }}=-0.822 \mathrm{~V}, \mathrm{I}=0.158 \mathrm{nA}$, total height $6.7 \AA$
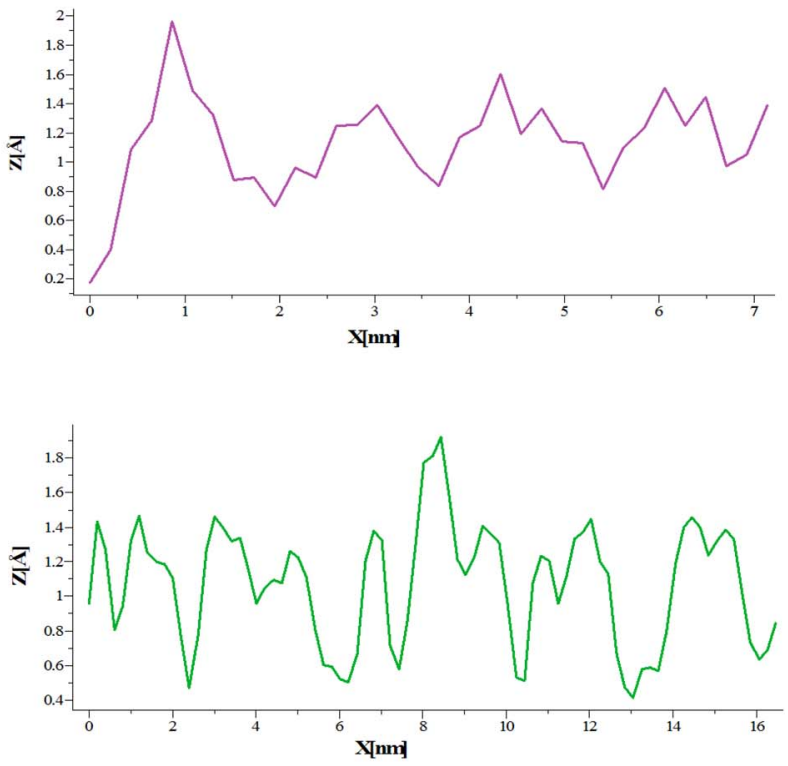

(b) line profiles; distance between peaks $15 \AA$ and $30 \AA$, respectively

FIG. 6. (Color online) STM topography of galvinoxyl on Au(111), acquired at $140 \mathrm{~K}$, sample preparation at room temperature, subsequently cooled down afterwards, frame size $70 \mathrm{~nm}$.

both directions we determine the dimensions for the unit cell: $15 \AA \pm 1.5 \AA$ by $7 \AA \pm 0.7 \AA$. The distance known from $\pi-\pi$ bonding in the range of $3.5 \AA-3.8 \AA$ is comparable to the distances between the bordering tert-butyl groups of the molecules. The distance we observe along the $c$ axis for the stacks of galvinoxyl is thus quite high, although it is probable that these intermolecular forces originate mainly from $\pi-\pi$ bonding between the carbon rings. Both hydrogen bonding and the weak van der Waals forces are also known to be responsible for the dominating intermolecular interactions compared to the forces occurring between substrate and surface.

Taking into account the most reasonable possibilities for the orientation of the molecule on the surface only one model matches well with the pattern and density observed, depicted in Fig. 8(b). The molecule is "standing" on the $\mathrm{Au}(111)$ with the two tert-butyl groups on position 3 and 4 (carbon atoms, see Fig. 2) at the surface. Of course a reverse symmetry is also thinkable. In our opinion it is impossible to decide from our data which model applies. The small shadows with a lower height visible in Fig. 8(b) could stem from a tilt of the molecule regarding the rectangular axis to the surface. This could be between 5 and 10 degrees. This is discussed in the context of the second configuration in Sec. III A 2.

\section{Configuration II}

Additionally to the first stable structure we observed a second one which occurred only after the sample had been cooled down to $40 \mathrm{~K}$ before. The configuration seems to be less influenced by the substrate structure than the first one.
Thus, the binding to the surface being probably weaker. Usually, the $22( \pm 1) \times \sqrt{ } 3$ reconstruction is observed to disappear, if a self-assembled layer is formed on $\mathrm{Au}(111)$. Only few opposite cases have been reported. ${ }^{35}$

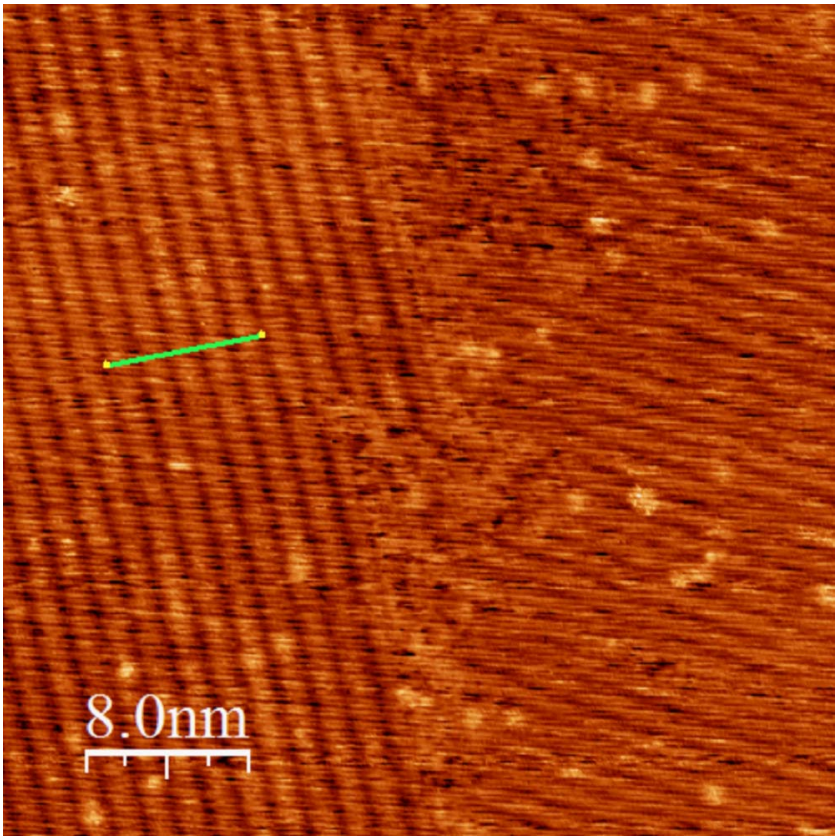

FIG. 7. (Color online) STM topography of galvinoxyl on $\mathrm{Au}(111)$ with more detailed resolution, acquired at $140 \mathrm{~K}$, sample preparation at room temperature, cooled down afterwards for the measurement, frame size $40 \mathrm{~nm}$. 


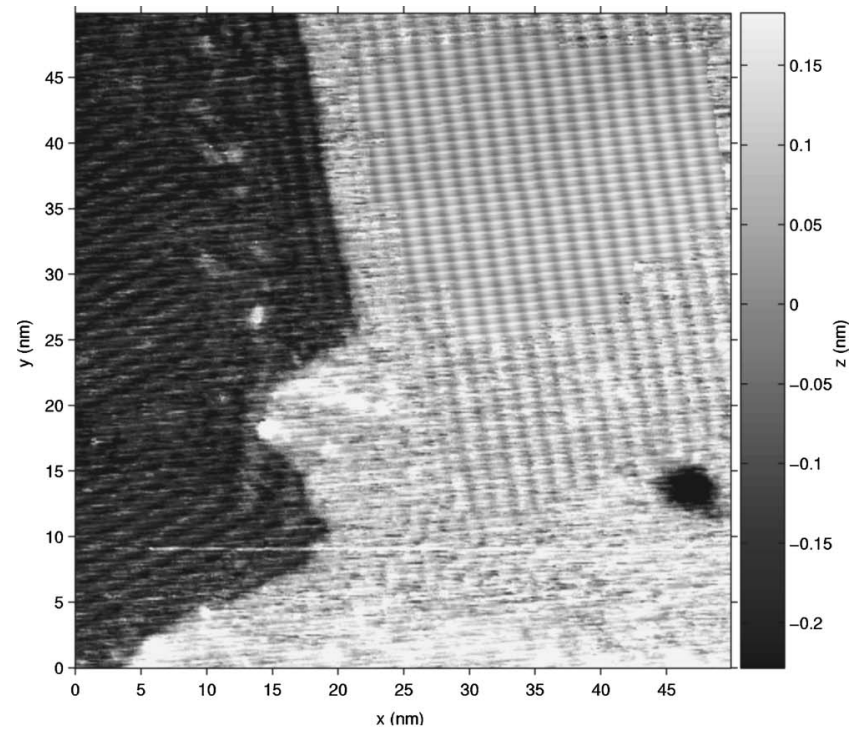

(a) Ugap $=-0.822 \mathrm{~V}, \mathrm{I}=0.158 \mathrm{nA}$, total height $8.6 \AA$

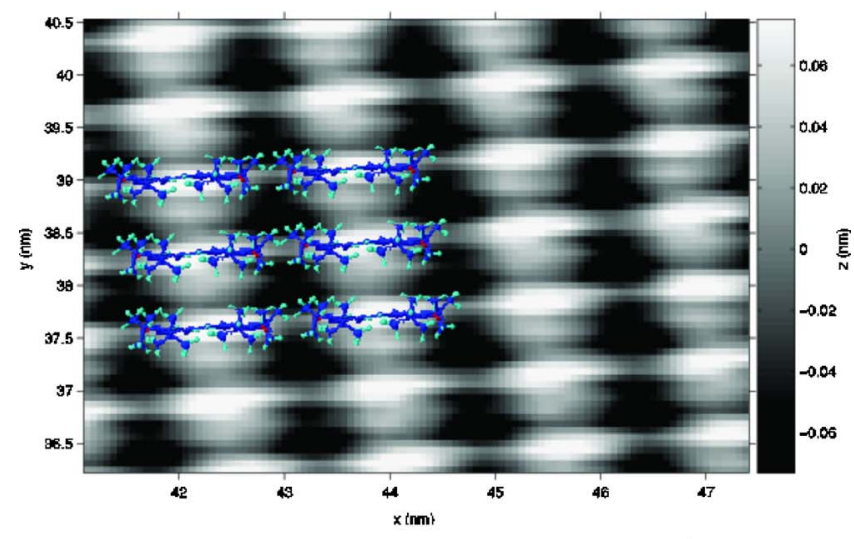

(b) Ugap $=-0.822 \mathrm{~V}, \mathrm{I}=0.158 \mathrm{nA}$, total height $6.7 \AA$

FIG. 8. (Color online) Galvinoxyl layer deposited at room temperature onto $\mathrm{Au}(111)$, measurement performed at $140 \mathrm{~K}$, preparation at room temperature, subsequently cooled down for the STM measurement. The area with reduced noise is correlation filtered (Ref. 41).

In the following we present an overview of the second structure observed with galvinoxyl on Au(111), deposition from ambient into UHV, using the same procedure as for the first configuration. We did not obtain a good resolution of this structure at $40 \mathrm{~K}$. This could be related, e.g., to a more unstable aggregate related to the phase transition as mentioned before (see Sec. I). It is known from the crystal structure that the properties change significantly below $85 \mathrm{~K}$. Consequently the properties like spin density, localization, and chemical bonding could be changed and can lead to difficulties in getting a good resolution in STM imaging. Also other authors describe surprising observations: As bulk and beyond the phase transition galvinoxyl appears to be a ferromagnet with an extrapolated Curie temperature of $19 \mathrm{~K}$. The nature of the transition at $85 \mathrm{~K}$ seems to be quite drastic in many respects. Probably it is not only magnetic, but accompanied with severe structural and electronic rearrangements. The heat capacity grows by a factor of 3 at this transition, ${ }^{42}$ and analysis of the crystal structure below $85 \mathrm{~K}$ failed due to disintegration of the crystals. ${ }^{26}$ The reason for the lowered distance from configuration I at $7 \AA$ to $5.4 \AA$ in configuration II could be different expansion coefficients of $\mathrm{Au}(111)$ and galvinoxyl. For gold it is $7.1 \times 10^{-5} \mathrm{~K}$ and for organic molecules roughly $7 \times 10^{-4} \mathrm{~K}$. The factor of about 10 could explain that at low temperature the molecules are more "concentrated" resulting in a lower stacking distance regarding the $c$ axis of the aggregate on the $\mathrm{Au}(111)$. If the sample is warmed up to room temperature the denser stacked arrays persist over time periods of at least days. Interestingly, so-called charge transfer configurations in neighboring pairs of galvinoxyl are considered as stable. ${ }^{19}$ Charge is then transferred from one to the other molecule and the molecules become partly ionic. It is tempting to attribute the bright spots in the topography of configuration II presented in Fig. 9 to such regions. However we must leave this undecided.

The second configuration is shown in Fig. 9. The average corrugation is slightly higher than before $(1.2 \AA$ compared to $0.8 \AA$ ) and a more complex pattern is observed. The angle between rows of molecules repeats every second pattern. An angle of 60 degrees is included by the stacks of molecules. In Fig. 9(b) the distance of $10.8 \AA \pm 1 \AA$ between the bright spots inside the stacks is shown. As we can see in the second profile only every second molecule has a bright spot in one row, the molecules are separated by a distance of $5.4 \AA \pm 1 \AA$ along the $c$ direction. The distance is slightly bigger than the distance of $3.785 \AA$ found for the crystalline phase ${ }^{3}$ along the $c$ axis, and slightly smaller than the distance of $7 \AA$ found in the first configuration. This might be due to the tilt angle of the standing molecule off the vertical axis being smaller or zero with this structure. Consequently, the positions of the galvinoxyl molecules deviate only very slightly from standing exactly 90 degrees upright on the $\mathrm{Au}(111)$ surface.

The reconstruction can be viewed with a better contrast for the diagonal lines in Fig. 10. The period we observe for the $\mathrm{Au}$ reconstruction is $63 \pm 0.5 \AA$, compatible with the $22( \pm 1) \times \sqrt{ } 3$ herringbone reconstruction. ${ }^{34,35,43}$ Surprisingly, this reconstruction is preserved with the molecular assembly on top. ${ }^{44}$

The zoom allows to see submolecular contrast of the configuration. The Fig. 11(a) with a frame size of about $11 \mathrm{~nm}$ shows rows with a pattern according to the herringbone structure including a 60 degree angle. The molecules are surrounded by alternating more and less bright spots at the endpoints. The apparent height of these bordering regions of the molecule is modulated inside the stacks.

The observed length of $1.53 \mathrm{~nm}$ in-between the bright spots fits well with the measured length of $\simeq 13.9 \AA$ inside one of the crystal configurations. ${ }^{25}$ The corrugation of the bright spots appears large, however this height modulation of $1.2 \AA$ within individual molecules is substantially smaller compared to the interlayer spacing of $\sim 5 \AA$ in the molecular crystal. The analysis of Fig. 11(b) reveals that in the profile always two high protrusions follow two low ones and so on. Always two very bright spots follow two weaker ones, so that every second molecule has either two weaker or two stronger bright spots. The molecules in-between have one 


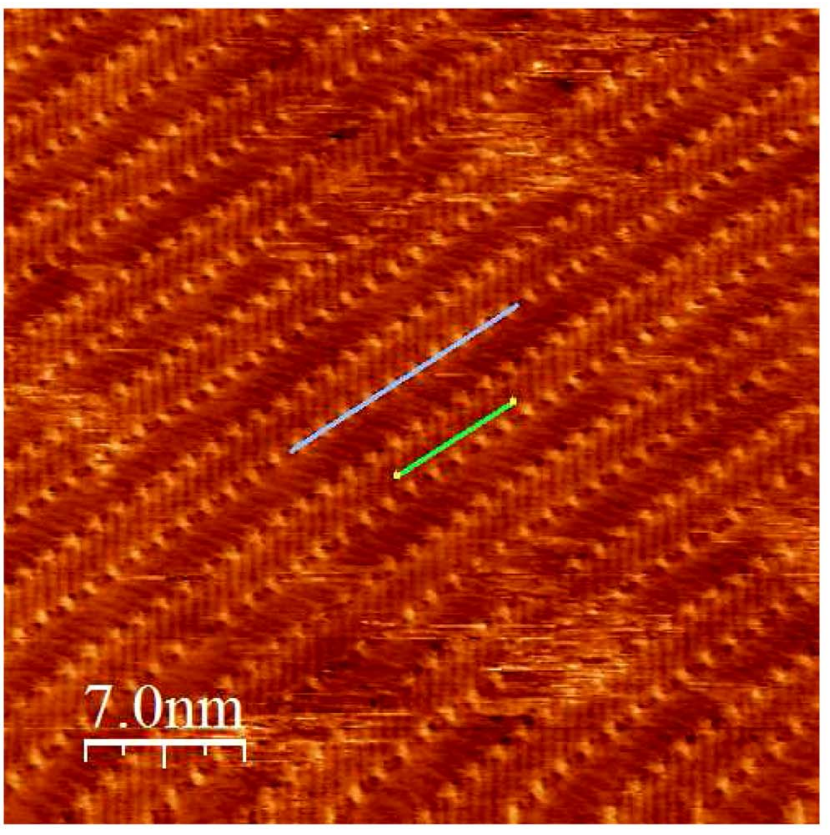

(a) $\mathrm{U}_{\text {gap }}=-0.800 \mathrm{~V}, \mathrm{I}=0.503 \mathrm{nA}$, total height $2,7 \AA$
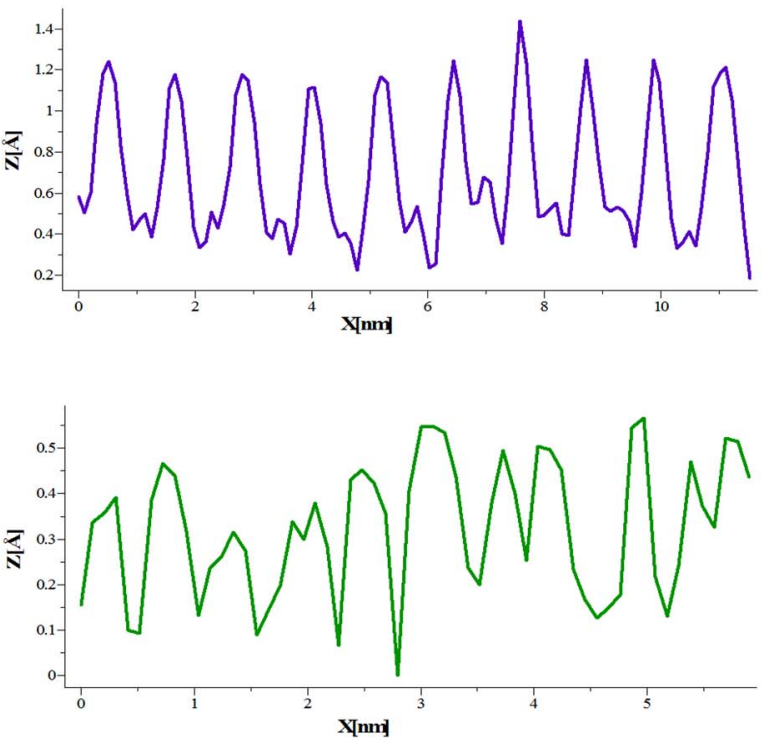

(b) line profiles with peaks periods corresponding to $10.8 \AA$ and $5.4 \AA$, resp.

FIG. 9. (Color online) STM topography galvinoxyl on $\mathrm{Au}(111)$, second configuration, molecules deposited at room temperature, sample cooled to $40 \mathrm{~K}$, STM measured at room temperature, frame size $35 \mathrm{~nm}$.

bright and one less bright spot. The tert-butyl parts of adjacent galvinoxyl molecules inside the stacks seem to be overlapping.

Figure 12 exhibits the same section with improved intramolecular resolution. The model pattern superimposed on the STM image indicates the orientation of the molecules inside the assembly. Two slightly different variations of the molecule structures are shown, one with absolute parallel molecules inside the stacks and one with alternating shifts along the lateral axis of the molecule. This model allows us to estimate the shift between the individual molecules along the lateral axis to about $2 \AA$. The highlighted side of each

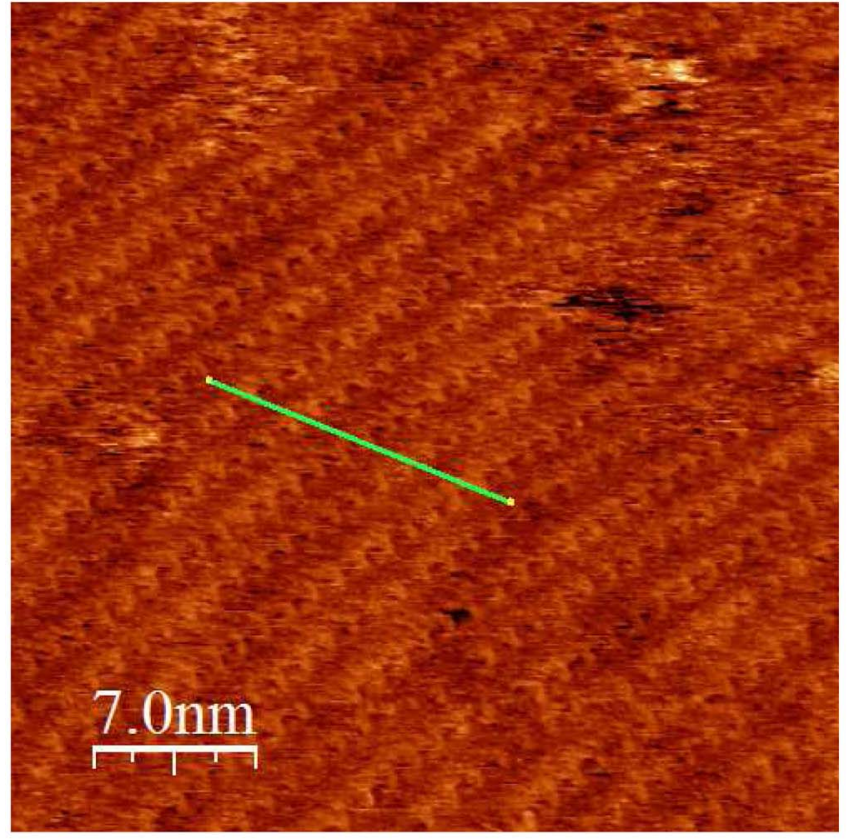

(a) $\mathrm{U}_{\text {gap }}=-0.638 \mathrm{~V}, \mathrm{I}=0.534 \mathrm{nA}$, total height $3 \AA$

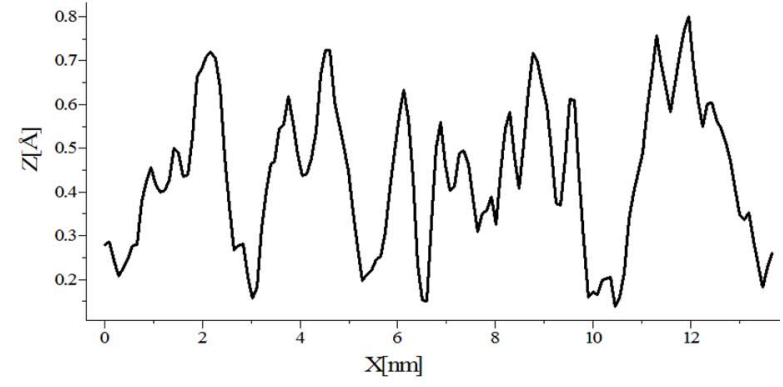

(b) line profile with a period corresponding to $63 \AA$

FIG. 10. (Color online) STM topography of galvinoxyl on the Au(111) surface showing the Au reconstruction below the adlayer, cooled to $40 \mathrm{~K}$, STM measurement acquired at room temperature, frame size $35 \mathrm{~nm}$. 


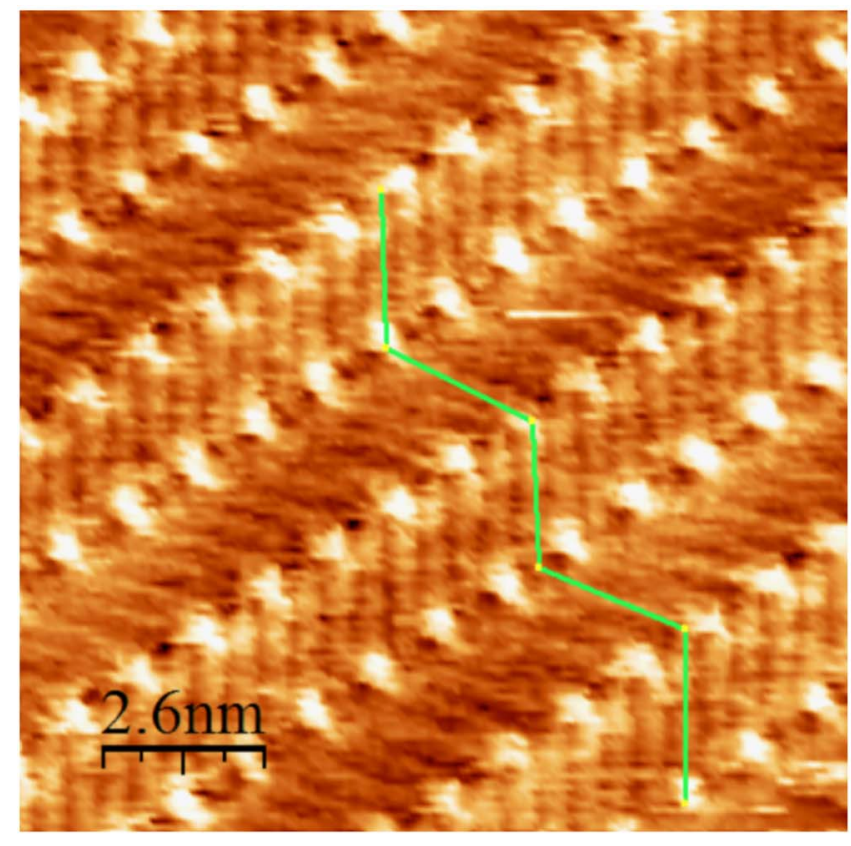

(a) $\mathrm{U}_{\text {gap }}=-0.800 \mathrm{~V}, \mathrm{I}=0.503 \mathrm{nA}$, total height $2.0 \AA$

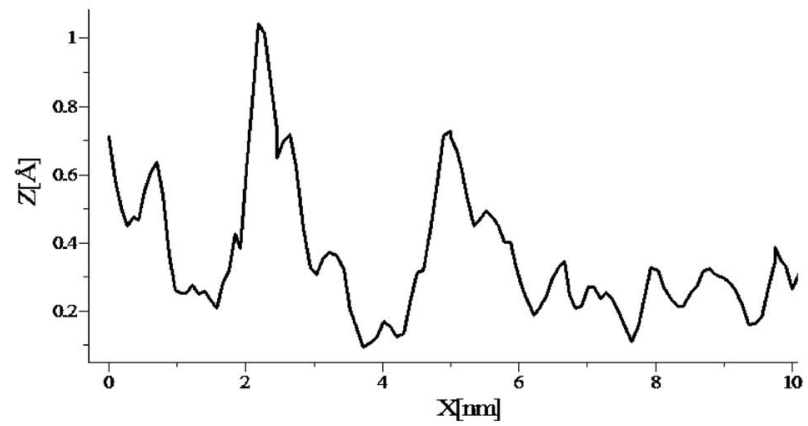

(b) line profile; distance between peaks corresponding to $22.5 \AA$

FIG. 11. (Color online) STM topography of galvinoxyl on Au(111), acquired at room temperature, frame size $11.1 \mathrm{~nm}$ by $11.4 \mathrm{~nm}$, cooled to $40 \mathrm{~K}$, STM images measured at room temperature.

molecule could be due to a different spin density. The shift of density can have different origin but in this case we can exclude a tip state change being responsible.

\section{B. EPR results}

EPR spectra of galvinoxyl in solution, galvinoxyl as a polycrystalline powder and galvinoxyl on an $\mathrm{Au}(111)$ substrate were measured. To obtain information about the isotropy, exchange frequency, and linewidth fits for the spectra were performed.

Although the conditions in UHV and ambient are not the same, we can assume a similar structure. First, the electrochemical measurements in Sec. III C confirmed a similar coverage and, second, STM under ambient conditions revealed a molecular adlayer. Additionally an Au(111) sample was covered with galvinoxyl in ambient conditions and afterwards measured in UHV. Similar structures could be observed. Although we could not achieve a high resolution, we could confirm that galvinoxyl forms a dense layer. The additional impurities from the ambient surrounding are negligible because they give no relevant EPR signal.

Figure 13 shows a $2.2 \mathrm{mM}$ solution EPR spectrum of galvinoxyl in ethanol at room temperature. It shows a doublet absorption centered at $g \simeq 2.00$. Similar patterns were shown by Scheffler et al. ${ }^{45}$ for a $0.01 \mathrm{mM}$ solution in an $\mathrm{O}_{2}$ free solvent like, for example, toluene. In the latter case a super-hyperfine splitting with 10 lines was resolved. ${ }^{46}$ The hyperfine splitting shown in Fig. 13 originates from the coupling of the single electron with the hydrogen proton at the methylcarbon at position 7 (see Fig. 2). The super-hyperfine splitting with the other protons is resolved in case of a very low concentration and an oxygen free solution. In the presented doublet the exchange interaction between the orbitals of the dissolved molecular oxygen and the orbitals of the single molecular electron in the free radical leads to a line broadening. The fit deducted by the blofit routine ${ }^{47}$ gives a hyperfine splitting constant of 5.57 Gauss, which is in agreement with values found in the literature. ${ }^{45,48}$

The EPR absorption spectrum obtained from the polycrystalline specimen shows a flat-topped single peak,

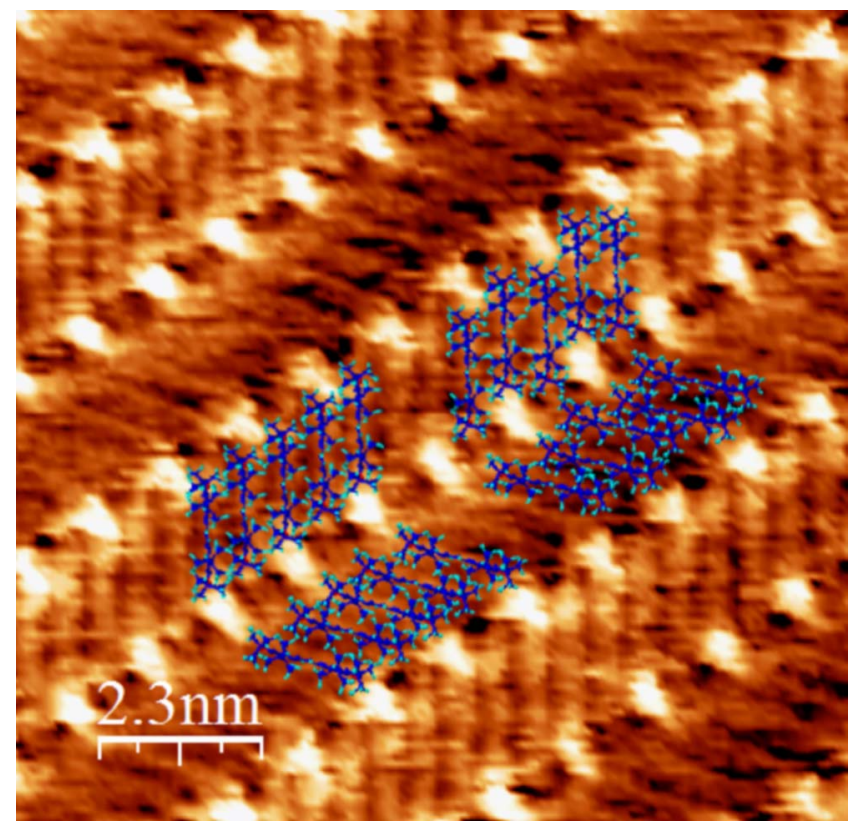

FIG. 12. (Color online) Structure models for configuration II. 


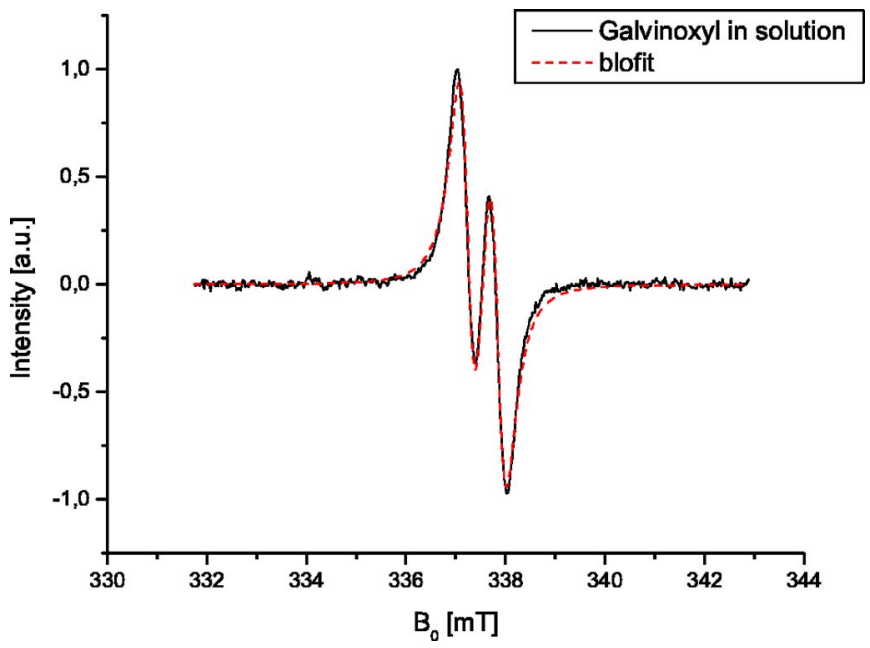

FIG. 13. (Color online) EPR measurement of galvinoxyl in ethanol solution (continuous black line) compared with the fitting obtained from blofit (dashed red line).

Lorentz-type adsorption with a width of 5.91 Gauss between points of maximum slope (derivative shown in Fig. 14), centered at $g \simeq 2.00$. The anisotropy characteristic and linewidth is comparable to the data of Mukai et al. ${ }^{48}$ The high spin exchange interaction between the singly occupied molecular orbitals (SOMO) leads to overlap of the super-hyperfine lines and narrowing of the spectral linewidth.

To measure EPR spectra of galvinoxyl on the $\mathrm{Au}(111)$ surface, the substrate was placed parallel to the magnetic field and the electric field as shown in the experimental section (Sec. II B). The spectrum shows a singlet with a linewidth of 8.1 Gauss, which exceeds the linewidth of the powder spectrum in accordance with the higher intermolecular distance inside the layer compared to the crystal.

The EPR measurements confirm that the radical galvinoxyl is not reduced upon binding to the surface but is stable and persistent as a free radical. Comparison of the solution and powder spectra with the spectrum of the single layer

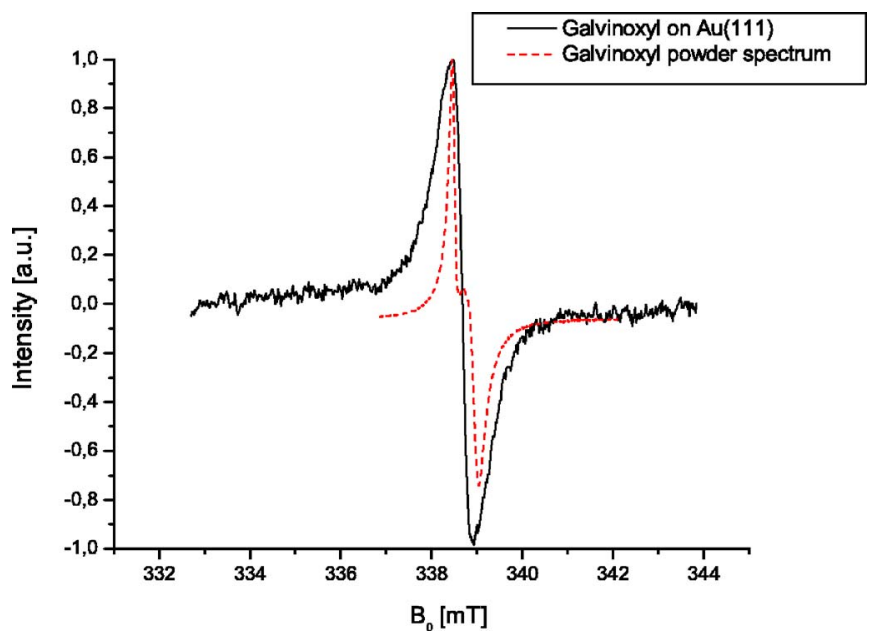

FIG. 14. (Color online) EPR measurement of galvinoxyl on the $\mathrm{Au}(111)$ (continuous black line) surface compared with the galvinoxyl powder spectrum (dashed red line). reveals that the exchange frequency $\omega_{\mathrm{ex}}$, which leads to an exchange narrowing of the absorption line, decreases in the order $\omega_{\mathrm{ex}}>\omega_{\mathrm{ex}}$ (surface layer) $>\omega_{\mathrm{ex}}$ (solution). An estimation of the exchange frequency using modified Bloch equations yields approximately $\omega_{\text {ex }}$ (surface layer) $\sim 1 \mathrm{GHz}$. If we assume an exchange through directly overlapping of the single electron orbitals, an upper limit of $11 \AA$ for the distance between the centers of highest spin density can be estimated. ${ }^{49}$

The same number of molecules on both the $\mathrm{Au}(111)$ layer and a glass substrate of the same size differ in their double integrated spectra: The signal of galvinoxyl on Au(111) is enhanced by a factor of 4 .

\section{Electrochemical measurements}

Additionally to the structural studies we studied the galvinoxyl bound to the $\mathrm{Au}(111)$ surface with cyclic voltammetry. For this purpose we used the cyclic voltammetry method in a setup described in the experimental section.

Cyclic voltammograms of the galvinoxyl coated gold electrode show well-defined cathodic and anodic peak currents. The linear variation of the peak currents with scan rate and the small peak-to-peak separation are typical characteristics of electroactive surface confined species [Figs. 15(a) and $15(\mathrm{~b})] .{ }^{50}$ The surface coverage $\Gamma=1.0 \times 10^{-10} \mathrm{~mol} \mathrm{~cm}{ }^{2}$ obtained from integration of the area under the voltammetric curve is less than $\Gamma=1.4 \times 10^{-10} \mathrm{~mol} \mathrm{~cm}^{2}$ and 2.0 $\times 10^{-10} \mathrm{~mol} \mathrm{~cm} \mathrm{~cm}^{2}$ reported for galvinoxyl anchored on a gold surface via a molecular spacer and spacer diluted with other molecules, respectively. ${ }^{51-53}$ In aqueous solution the oxidation of the surface bound galvinoxyl is coupled to the loss of a proton as indicated by the linear dependence of $E^{0}$ from $p \mathrm{H}$ with a slope of $-54 \mathrm{mV} / \mathrm{pH}$ [see Fig. 15(c)]. Proton coupled electron transfer is important in many processes between surface and the adsorbed molecules, in this case the galvinoxyl. According to the voltammetric response one could find a linear plot regarding $I$ versus $V$ in both directions. Appropriately to the Nernst equation, a plot of $E$ versus $p H$ should give a straight line as we observed in Fig. 15(b). This suggests a one-proton one-electron process while reducing and oxidizing the molecule. The plot of $E^{0}$ as a function of $p \mathrm{H}$ shows a slope of $-54 \mathrm{mV} / p \mathrm{H}$ (Fig. 15, III). This value is close to the slope of $E^{0}$ vs $p \mathrm{H}$ plot, i.e., $-59 \mathrm{mV} / p \mathrm{H}$, for the one-electron one-proton process showing a Nernstian dependence. $^{50}$ The same dependence with a slope of $-60 \mathrm{mV} / p \mathrm{H}$ was reported for galvinoxyl anchored on a gold surface via spacer. ${ }^{54}$

We used the herein obtained results to recalculate the coverage. Compared to the $1.0 \times 10^{-10} \mathrm{~mol} \mathrm{~cm}{ }^{2}$ derived by cyclic voltammetry we found with STM a slightly higher coverage of $1.57 \pm 0.16 \times 10^{-10} \mathrm{~mol} / \mathrm{cm}^{2}$, respectively, $9.46 \pm 0.94 \times 10^{13}$ molecules per square $\mathrm{cm}$ for configuration I. This corresponds to 0.068 molecules per $\mathrm{Au}$ atom compared to 0.059 molecules per $\mathrm{Au}$ atom calculated for the second configuration with a coverage of $1.37 \pm 0.16$ $\times 10^{-10} \mathrm{~mol} / \mathrm{cm}^{2}$, respectively, $8.23 \pm 0.82 \times 10^{13}$ molecules per square $\mathrm{cm}$ as determined from our STM measurements.

The electrochemical data indicate that the galvinoxyl is organized in a dense layer and suggest that a monolayer 


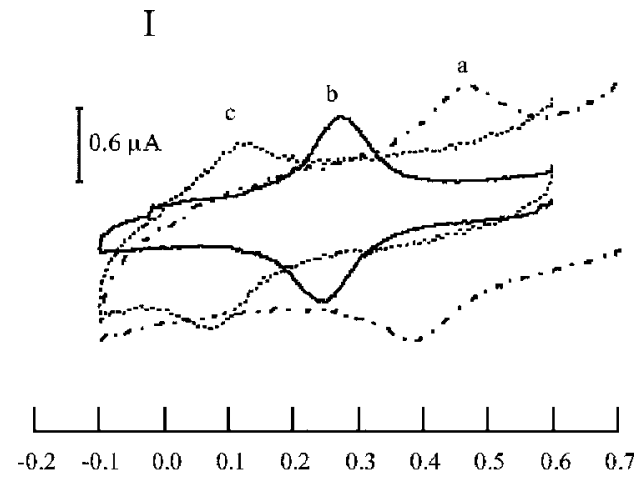

$\mathrm{E} / \mathrm{V}$ vs $\mathrm{Ag} \mid \mathrm{AgCl}$

(a) $\mathrm{CV}$ of galvinoxyl-modified gold electrode in $0.1 \mathrm{M} \mathrm{Na}_{2} \mathrm{HPO}_{4}$ at $\mathrm{pH}: 4.6(\mathrm{a}) ; 7.8$ (b) and 10.4 (c)

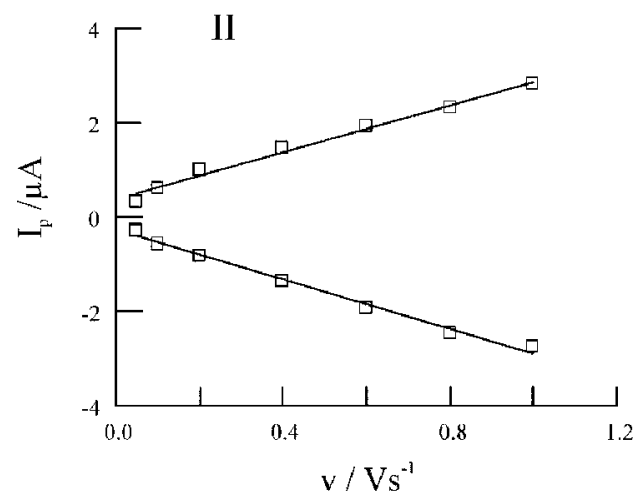

(b) Plot of $I_{p}$ versus scan rate form CV at $\mathrm{pH} 7.8$

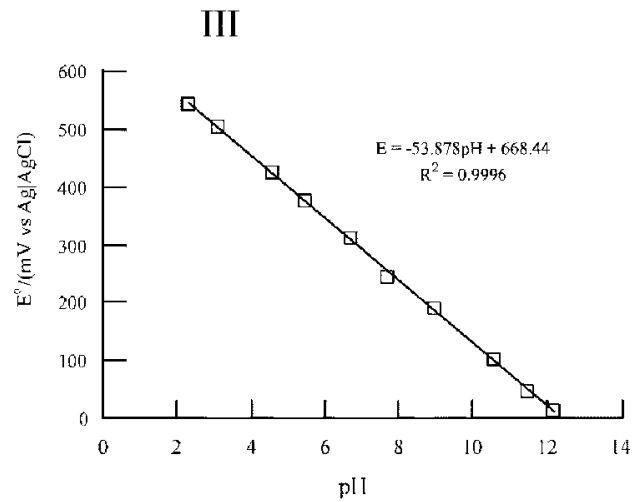

(c) Plot of $E^{0}$ versus $\mathrm{pH}$ from CVs shown in I and others

FIG. 15. Voltammetry measurements.

is formed like in UHV, possibly with additional clusters aggregated preferentially at the step edges of the surface.

\section{Summary}

The galvinoxyl molecule forms at least two different types of molecule assemblies on the $\mathrm{Au}(111)$ surface. The first configuration is more similar to the structure in galvinoxyl crystals. The second configuration occurs with cooling down the sample to $40 \mathrm{~K}$, which suggests relative size of unit cells playing a role. In both cases a correlation between the sixfold symmetry of the $\mathrm{Au}(111)$ top layer and the arrangement of the galvinoxyl molecules is present. Consequently the substrate structure is relevant for the assembling, although the binding to the surface is estimated to be quite weak. Probably the whole assembly is only connected through hydrogen bonds or through the weak van der Waals interaction. In contrast to configuration II, with configuration I no herringbone reconstruction is observed. It is however not clear that the $\mathrm{Au}(111)$ reconstruction is lifted with configuration I. The reconstruction could remain invisible in the STM topographies. Note, such sublayer contrasts are difficult to achieve and depend delicately on tip state and tunnelling parameters.

The second structure observed after cooling down to $40 \mathrm{~K}$ gives a density of $8.23 \times 10^{13}$ molecules per $\mathrm{cm}^{2}$. The distance between the molecules along the $c$ axis decreases after the sample had been cooled down. The reason could be the factor of 10 between the expansion coefficients of the $\mathrm{Au}(111)$ surface and the galvinoxyl molecules. This energy configuration change is strong enough to persist upon achieving room temperature and time.

The protruding dots in the topography shown in Fig. 11 with two high peaks in a row could be explained by alternate swapping of the galvinoxyl within the stacks. It is assumable that always two molecules in a row shown in the line section stand on the surface like in configuration I and the next two are flipped. Additionally a localization of the single spin orbitals could occur related to an eventual ferromagnetic interaction. Exchange integral and overlap integral depend decisively on the intermolecular distance and orientation, i.e., the lattice parameters of the aggregate. Small lattice constants, associated with large SOMO-SOMO overlap, would favor antiferromagnetic coupling or even formation of a molecular bond, while larger lattice constants with small but appreciable overlap rather favor ferromagnetic interaction. ${ }^{19}$

We tried to estimate the signal intensity of the galvinoxyl on the $\mathrm{Au}(111)$ surface compared to the number of spins. The signal intensity for the molecular layer on $\mathrm{Au}(111)$ is 4 times higher than expected. This may point towards the galvinoxyl layer on the $\mathrm{Au}(111)$ surface being ferromagnetic.

The CV results show a slightly lower concentration (about $20 \%)$ than inferred for the first configuration. This can be due to a higher density of impurities expected for the gold electrode surface, which leads to an effectively smaller surface area for adsorption.

\section{CONCLUSION}

We have found galvinoxyl self-assembles as monolayer on $\mathrm{Au}(111)$. Two different configurations occur, one with a unit cell of $15 \AA$ by $7 \AA$ and the other one with $22.5 \AA$ by $5.4 \AA$. The configurations correspond to coverages of 0.068 and 0.059 galvinoxyl molecules per Au atom, respectively, and we suggested structure models for both configurations.

EPR measurements confirmed the galvinoxyl not being reduced on the $\mathrm{Au}(111)$ surface and remaining stable as radical. An estimation of the exchange frequency of 
$\sim 1000 \mathrm{MHz}$ yields a more dense packing on the surface than in the crystal, involving orbitals from the missing third dimension into the exchange interaction. The distance between the single spin orbitals could be estimated to be smaller than $11 \AA$ along the $c$ axis.

The electrochemical results suggest the formation of an electroactive monolayer upon exposure of the gold electrode to galvinoxyl solution. The monolayer shows the oneelectron one-proton transfer Nernstian behavior and the molecule density on the surface $\left(\Gamma=6.022 \times 10^{13}\right.$ molecule $\mathrm{cm}^{2}$ ) is compatible with the STM topographies.

\section{ACKNOWLEDGMENTS}

This work was supported by Ministry of Sciences and Culture of Lower Saxony through the Ph.D. program Synthesis and Characterisation of Surfaces. Further support was provided by NanoNed, the Dutch nanotechnology programme of the Ministry of Economic Affairs, and by the EU project ASPRINT. The authors wish to acknowledge stimulating discussion with Leroy Cronin, Peter Jaitner, Gilles de Wijs, Rob A. de Groot, Jisk J. Attema, and Emiliano Feresin.
*URL: http://www.evsf2.science.ru.nl/sylvia/; Electronic address: s.speller@science.ru.nl

${ }^{1}$ F. Schreiber, Prog. Surf. Sci. 65, 151 (2000).

${ }^{2}$ K. Mukai, Bull. Chem. Soc. Jpn. 42, 40 (1969).

${ }^{3}$ D. E. Williams, Mol. Phys. 16, 145 (1969).

${ }^{4}$ C. Durkan and M. E. Welland, Appl. Phys. Lett. 80, 458 (2001).

${ }^{5}$ J. L. Pitters, P. G. Piva, X. Tong, and R. A. Wolkow, Nano Lett. 3, 1431 (2003).

${ }^{6}$ N. P. Guisinger, R. Basu, and A. S. B. M. C. Hersam, Ann. N.Y. Acad. Sci. 1006, 227 (2003).

${ }^{7}$ M. E. Greene, N. P. Guisinger, R. Basu, A. S. Baluch, and M. C. Hersam, Surf. Sci. 559, 16 (2004).

${ }^{8}$ A. R. Alemozafar and R. J. Madix, J. Phys. Chem. B 109, 11307 (2005).

${ }^{9}$ Y. L. Chan, W. W. Pai, and T. J. Chuang, J. Phys. Chem. B 108, 815 (2004).

${ }^{10}$ W. W. Pai, Y. L. Chan, and T. J. Chuang, Chin. J. Phys. (Taipei) 43, 212 (2005)

${ }^{11}$ W. W. Pai, Z. Zhang, J. Zhang, and J. F. Wendelken, Surf. Sci. 393, L106 (1997).

${ }^{12}$ N. Pertaya, Ph.D. thesis, Department of Physics of the Free University Berlin, 2004, Chap. 3.

${ }^{13}$ D. Matsushitaa, H. Ikedaa, A. Sakaia, S. Zaimab, and Y. Yasuda, Thin Solid Films 369, 293 (2000).

${ }^{14}$ D. Matsushita, H. Ikeda, A. Sakai, S. Zamai, and Y. Yasuda, Jpn. J. Appl. Phys., Part 1 40, 2827 (2001).

${ }^{15}$ N. Takehiro, F. Besenbacher, E. Laegsgaard, K. Tanaka, and I. Stensgaard, Surf. Sci. 397, 145 (1998).

${ }^{16}$ M. Matsumoto, K. Fukutani, T. Okano, K. Miyake, H. Shigekawa, H. Kato, H. Okuyama, and M. Kawai, Surf. Sci. 454-456, 101 (2000).

${ }^{17}$ B. Hammer, Surf. Sci. 459, 323 (2000).

${ }^{18}$ S. J. Blundell and F. L. Pratt, J. Phys.: Condens. Matter 16, R771 (2004).

${ }^{19}$ M. Kinushita, Jpn. J. Appl. Phys., Part 1 33, 5718 (1994).

${ }^{20}$ G. M. Coppinger, J. Am. Chem. Soc. 79, 501 (1957).

${ }^{21}$ J. J. Windle and W. H. Thurston, J. Chem. Phys. 27, 1429 (1957).

${ }^{22}$ F. Dietz, N. Tyutyulkov, and M. Baumgarten, J. Phys. Chem. B 102, 3912 (1998).

${ }^{23}$ S. Luo and K. Yaoa, J. Magn. Magn. Mater. 257, 11 (2003).

${ }^{24}$ K. Awaga, T. Sugano, and M. Kinoshita, J. Chem. Phys. 85, 2211 (1986).

${ }^{25}$ K. Mukai and A. Sogabe, J. Chem. Phys. 72, 598 (1980).

${ }^{26}$ K. Chi, J. Calabrese, J. Miller, and S. Khan, Mol. Cryst. Liq.
Cryst. 176, 185 (1989)

${ }^{27}$ P. D. Bartlett and T. Funahashi, J. Am. Chem. Soc. 84, 2596 (1962).

${ }^{28}$ Q. Guo, G. Rimbach, H. Moini, S. Weber, and L. Packer, Toxicology 179, 171 (2002).

${ }^{29}$ Parcom, Parker Instruments Inc., available at http:// www.parcom.com (2001).

${ }^{30} \mathrm{~N}$. Niermann et al. (unpublished).

${ }^{31}$ E-LINE EPR spectrometer system, technical manual, Varian Report No. 87-125-102, d473 ed., 1971.

${ }^{32}$ T. H. Degefa, P. Schön, D. Bongard, and L. Walder, J. Electroanal. Chem. 574, 49 (2004).

${ }^{33} \mathrm{H}$. by Hyperchem, Hyperchem, available at http:// www.hyper.com (2003).

${ }^{34}$ N. Takeuchi, C. T. Chan, and K. M. Ho, Phys. Rev. B 43, 13899 (1991).

${ }^{35}$ B. Hulsken, J. W. Gerritsen, and S. Speller, Surf. Sci. 580, 95 (1987).

${ }^{36}$ J. A. A. W. Elemans, M. C. Lensen, J. W. Gerritsen, H. van Kempen, S. Speller, R. J. M. Nolte, and A. E. Rowan, Adv. Mater. (Weinheim, Ger.) 15, 2070 (2004).

${ }^{37}$ S. Ceballos, G. Mariotto, S. Murphy, and I. Shvets, Surf. Sci. 523, 131 (2003).

${ }^{38}$ T. K. Yamada, Ph.D. thesis, Radboud University of Nijmegen, 2005.

${ }^{39}$ G. Grampp, S. Landgraf, K. Rasmussen, and S. Strauss, Spectrochim. Acta, Part A 58, 1219 (2001).

${ }^{40}$ J. Gomes and J. Gomes, J. Mol. Struct.: THEOCHEM 503, 189 (2000).

${ }^{41}$ B. Hulsken, J. Gerritssen, and S. Speller (unpublished).

${ }^{42}$ K. Awaga, T. Sugano, and M. Kinoshita, J. Chem. Phys. 87, 3062 (1987).

${ }^{43}$ C. Wöll, S. Chiang, R. J. Wilson, and P. H. Lippel, Phys. Rev. B 39, 7988 (1989).

${ }^{44}$ In contrast to configuration II, with configuration I no herringbone reconstruction is observed. It is however not clear whether the $\mathrm{Au}(111)$ reconstruction is lifted with configuration I. The reconstruction could be invisible in the STM topographies. Note, such sublayer contrasts are difficult to achieve and depend delicately on tip state and tunnelling parameters.

${ }^{45}$ K. Scheffler and H. B. Stegmann, Elektronenspinresonanz, Organische Chemie in Einzeldarstellungen, Band 12 (SpringerVerlag, Berlin, 1976).

${ }^{46}$ E. Müller, K. Ley, K. Scheffler, and R. Mayer, Chem. Ber. 91, 
2682 (1958).

${ }^{47}$ H.-J. Steinhoff (unpublished).

${ }^{48}$ K. Mukai, K. Ueda, K. Ishizu, and Y. Deguchi, J. Chem. Phys. 77, 1606 (1982).

${ }^{49}$ G. Jeschke, Rapid Commun. Mass Spectrom. 23, 227 (2002).

${ }^{50}$ A. J. Bard and L. R. Faulkner, Electrochemical Methods; Fundamentals and Application (Wiley, New York, 1980).
${ }^{51}$ H. O. Finklea and R. M. Haddox, Phys. Chem. Chem. Phys. 3, 3431 (2001).

${ }^{52}$ D. A. Shultz and G. N. Tewt, J. Org. Chem. 59, 6159 (1994).

${ }^{53}$ T. Sagara and T. Midorikawa, Langmuir 14, 3682 (1998).

${ }^{54}$ R. M. Haddox and H. O. Finklea, J. Electroanal. Chem. 550-551, 351 (2003). 